\title{
Automatic Text Summarization for Code- Mixed Language using Fuzzy Logic
}

\author{
Madhuri A. Tayal', Animesh Tayal ${ }^{2}$ and Pratibha Kokardekar ${ }^{3}$ \\ ${ }^{1}$ Assistant professor, Shri Ramdeobaba College of Engineering and Management, \\ Nagpur. Information Technology Department, India \\ ${ }^{2}$ Assistant professor, S.B. Jain Institute of Technology, Management and Research, \\ Nagpur. CSE department. \\ ${ }^{3}$ Assistant professor, Shri Ramdeobaba College of Engineering and Management, \\ Nagpur. Information Technology Department.
}

\section{ABSTRACT}

Natural Language Processing (NLP) is a domain of computing and natural linguistics. It is related to the unique conversation among machines and languages used by human. We are leaving in the era of machines, data and intelligence where the amount of information on the Internet is raising continuously, the method which are competent to build it preserved for users, e.g., by text summarization, question answering chat bots etc. The difficulty is highlighted with news stories, where numerous news providers account on same measures by same facts. Application like Automatic text summarization can resolve this challenge. By creating a wide-ranging summary of a given set of documents, Summarization can deal with the problem of information load through retrieving and showing the main content. This is still increased via the escalating quantity of information in community media where collection opinion in diverse languages can be verified. This paper addresses the boundaries of summarization by means of upward techniques that work in an extremely tall quantity and for multilingual scenario. Still the evaluation of automatically formed summaries in diverse languages is a exigent problem for the summarization community, as because human hard work are multiplied to make model summaries for each language. The retention ratio for above method is found to be around 75\%.

KEY WORDS: NLP, POS-TAGGER, SUMMARY, TEXT DOCUMENT, EVALUATION, FUZZY LOGIC.

\section{INTRODUCTION}

World Wide Web is constantly generating content which is in many languages (code-mixed). Such data is difficult to handle, language detection and translation of these text fragments have become an extremely important

\section{ARTICLE INFORMATION}

${ }^{*}$ Corresponding Author: tayalma@gmail.com

Received 14th Oct 2020 Accepted after revision 28th Dec 2020

Print ISSN: 0974-6455 Online ISSN: 2321-4007 CODEN: BBRCBA

Thomson Reuters ISI Web of Science Clarivate Analytics USA and Crossref Indexed Journal

\section{1) Clarivate $\begin{gathered}\text { Analytics } \\ \text { rossef }\end{gathered}$}

NAAS Journal Score 2020 (4.31)

A Society of Science and Nature Publication,

Bhopal India 2020. All rights reserved.

Online Contents Available at: http//www.bbrc.in/

Doi: $h t t p: / / d x$.doi.org/10.21786/bbrc/13.14/36 problem. Many efforts have been done in one language like [Madhuri. A. Tayal, 2017]. This approach was applied for English language. In this approach title of the document [Madhuri. A. Tayal, 2013] was identified, syntax analyzer was built [Madhuri. A. Tayal, 2014] as well as semantic representation is done. This method uses semantic sentence and word similarity method [Madhuri. A. Tayal, 2014] as basis for text summarization. The above method was also applied for toddler stories [Madhuri. A. Tayal, 2018].

The development of social media texts such as chats, micro-blogs, Whatsapp, and blogs has formed a lot of novel opportunities for information retrieval and language technology, but also numerous novel challenges. Codemixed data is the data which is in two or more languages 
and the source language for code-mixed data is free order as it is the user generated text. Whereas, the target language for this data is fixed order. Challenges in code-mixed data may occur due to contractions and transliteration. The existing language identification and translation systems are not intended to transaction with code-mixed text. [Ben King, 2013]

Codemixed text over social media may be in any language. The most common languages used in India are english and hindi. It is very difficult to process such kind of data. Currently, challenges faced are Handling the syntax of such sentence for detecting the languages, translation of such kind of sentences into one fixed languageso that it can further be processed for summarization.

\section{Types of Summarization:}

- Abstractive Text Summarization [Atif Khan, 2016]: This method builds an internal semantic demonstration and later uses natural language production technique to generate a summary. This summary is nearer to what an individual might express.

- Extractive Text Summarization [Tanveer Siddiqui]: This method retrieves the resultant output based on title, keywords, cue words and TF-IDF etc. from the original document.

- Hybrid Text Summarization: Using extractive summarization techniques, as well as abstractive methods it selects salient codes from the input reviews and embeds them into an automatically generated summary.

Literature Review: Worldwide utilization of computer mediated communication like Email, chats and most in recent times used, on social media like facebook and twitter has verified that code-mixed data is reasonably prevalent to the internet. In case of social-media data, there are various complications due to contradictions, non-standard spellings and structure as well as mixing of scripts. Many languages that use non-roman scripts like Hindi, Chinese, Bangla, and Arabic are often present in a romanized form. [G. Rohini, 2014], Very less work has been done on computational models of code-mixing due to deficiency of code-mixed data in conventional text corpora which makes data intensive methods hard to apply. To deal with the identification and labeling of the parts of text which are in different languages remains the first step, POS tagging and normalization remain the hard part to solve. There are existing methods, one of the challenging tasks is to identify the language. Spandana Gella and team have tried language identification task for short code-mixed documents containing one or two languages. [Spandana gella, 2014]

\section{Angla Bharti and CDAC [RMK Sinha, 1995].}

Anglabharti is an Indian machine translation system was developed by IIT, Kanpur under Prof. R M K Sinha. It uses a pattern directed approach using context free grammar like structures. The stratergy used in this lies between the transfer and interlingual approach. We referred this system but we cannot use this as Anglabharti supports translation from English to Indian languages and our work requires translation aid from transliterated hindi to English language [U. Ahmed, 2011] C-DAC has solutions for conversion of English to Hindi and other Indian languages like Assamese, Malayalam, Nepali, Bangla, Punjabi, Telugu and Urdu. A number of solutions have been developed in this area including Mantra, AnglaBharati and MaTra. Some of these are developed as part of multi institution consortium projects. These systems iffer in their underlying approach to translation as well as in language pairs and domains supported. The problem arrived to use this was same as this doesn't support translation from hindi to English.

2. Text Rank [Rada Mihalcea, 2004] [G. Rohini, 2014]: Text Rank is a graph based ranking algorithm. It runs on graph which is specially made for NLP application.e.g. key phrases etc.

3. Text as a Graph: It Identifies text units that best describe the task at hand, and add them as vertices in the graph. Identify relations that connect such text units, and use these relations to draw edges between vertices in the graph. Edges can be directed or undirected, weighted or unweighted. Until convergence iterate the graph-based ranking algorithm, sorts vertices based on their final score.

4. Cluster based method [Ganpathiraju,2002]: Clusters for summarizing has proved efficiency and gained popularity the principle behind similarity-based summarization is that important information is repeated in different sentence on the same event. Two features, verb similarity and noun similarity, are computed over the two primitive types, ranking of clusters would be done using tf-idf.

5. Fuzzy Logic [F. Kiyoumarsi, 2010]: Fuzzy logic is an extractive approach. This method extracts the sentences on the basis of its importance in the paragraph defined by the fuzzy analyzers. In this the importance of sentence is classified as the truth value with may have a real value between 0 and 1 . The process is:

1. Fuzzify all the input sentences into fuzzy membership parameters.

2. Applying all the rules to generate the fuzzy output functions.

3. Defuzzification of fuzzy output functions to get crisp output values.

4. It was only for single language.

6. Vertex Cover Algorithm [Ansamma John, 2015]: This algorithm represents division of its vertices which can cover up all the edges of the graph. Each vertex of the graph will represent a sentence from a document. Each edge is to be maintained when two sentences share some similarity and i. e through combined cosine similarity and Normalized Google distance measures12. This algorithm is applied on this graph which finds the vertexes having 
maximum information compared to others. Then according to score the sentences are incorporated in the final summary.

\section{METHODOLOGY}

In This method Domain Specific Input is used. CodeMixed strings are taken as input. These strings are then checked or Hinglish(English+ Hindi) sentences. If there are no Hinglish sentences, it collects the string and it is directly used as final output. But, if there are Hinglish sentences, they are separated into English and Hindi Strings. This separation is done using wordnet and shabdhkosh. Hindi String is passed for transliteration. Using the translation API the Hindi word is converted to English. It is then concatenated with the English string and a new sentence is generated with the same meaning. This new sentence is again checked for Hinglish words. If any word is found then it goes through the same process. If not, then it is kept as final output.

Dataset: The dataset is domain specific that is the information in the input text will just concentrate on one particular area. For which two domains of five hundred sentences are created i.e. Knowledge and Art.This will contribute towards better summarized text.

\section{for example:}

\section{Code-Mixed Text}

Nowadays बच्चों के लिये free education provide होती है This is very useful उनका future build करने के लिये|

Many students उसके advantages ले रहे है|

2.Translated Text: Nowadays kids are provided with free education provide. This is very useful for building their future . Many students take these advantages.

3.Summarized Text: Free education provided for kids builds their future and provide advantages to many of them. The elaborated method explained below in figure-1.

The input that is the source language is free order (codemixed of any two languages) and the output desired is fixed order (any one language among the two). Graphical User Interface was created using Tinkter. Python programming was done in Anaconda Spyder IDE. We have used Spyder i.e. statistical calculating environment through the sustenance of current libraries such as Pandas, NumPy, SciPy, or matplotlib etc. The process is explained here in detail.

Step-1 Translation Process: Translation was done by using Googletrans which is a free and limitless python library that implemented google translate API. This uses the Google Translate Ajax API to make calls to such methods as detect and translate. The maximum character limit on a single text is 15k. Stepwise one of the sample outputs is shown at the end of this paper.

\section{Features of Googletrans include}

1. Auto language detection
2. Fast and reliable-it uses the same servers that translate.google.consumes

3. Bulk translations

4. Customizable service URL.

5. Connection pooling (the advantage of using requests session).

Figure 1: Summarization Process Using Fuzzy Logic

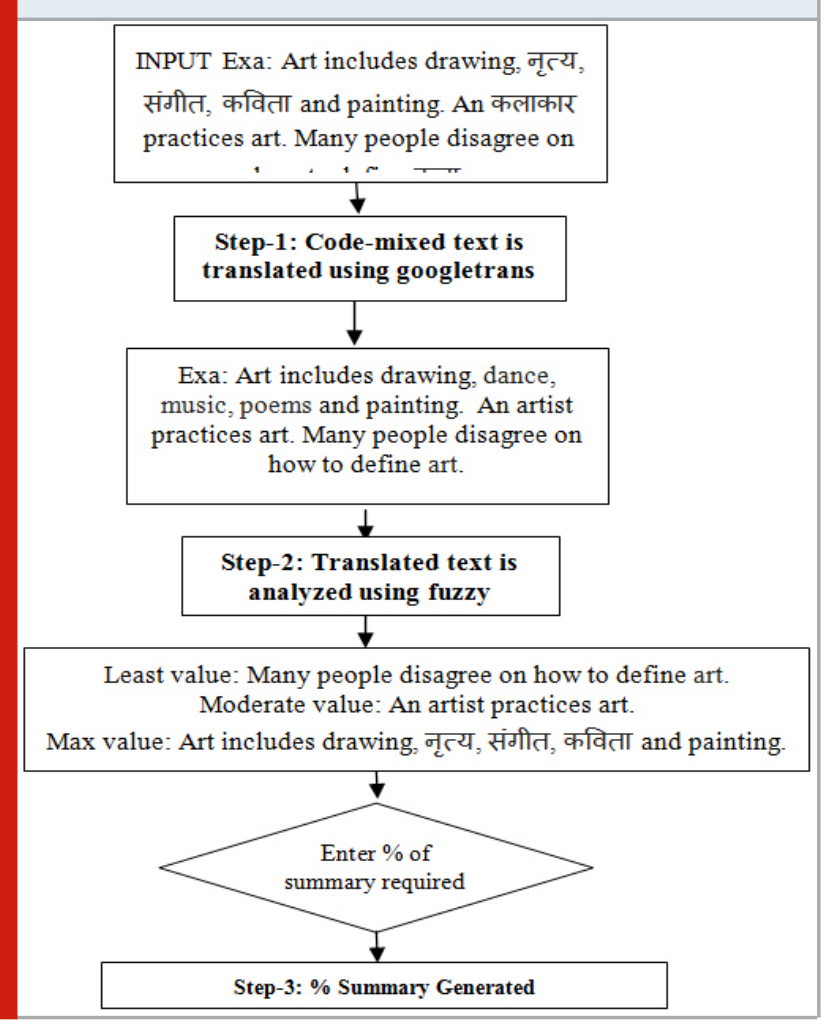

Step-2: Summarization Process: The text summarization system takes a domain specific input and processes it by applying fuzzy analyzers. Fuzzy analyzers used are

- The number of important thematic words and keywords in the sentence.

- Whether the sentence is first, middle or last sentence. (i.e. is the location of the sentence).

- This gives the priority of the sentences based on the logical parameters. Then the extracted text obtained by applying fuzzy analyzers gives the output summary.

\section{- Importance of Using the Fuzzy Analyzers:}

1. Keywords: In a summary there are certain keywords that are used a greater number of times and have high weightage in the paragraph. Also, there words that are relatively important based on the domain of the paragraph.

2. Location: Also, the position of the sentence helps to prioritize sentence as in a summary the last sentence plays an important role as it gives the gist of and has a higher precedence than middle sentences. Also, the first 
sentence is more important than the middle sentence as it gives the idea about the paragraph.

Table 1. Categorization of words according to keyword and location

\begin{tabular}{|l|c|c|c|}
\hline \multicolumn{1}{l|}{ Location } & \multirow{2}{*}{ LAST } & MIDDLE & START \\
\cline { 1 - 3 } Keyword & & & \\
\cline { 1 - 3 } LEAST.IMP & BETTER & GOOD & BETTER \\
\hline MODERATE.IMP & BETTER & GOOD & BETTER \\
\hline MAXIMUM.IMP & BEST & BETTER & BEST \\
\hline
\end{tabular}

In the above table 1, Input taken is keywords and location. Keyword has been categorized as least important, moderately important and max important. Location has been categorized as last, first and middle sentences. These parameters have been assigned a crisp input values so that they can produce crisp output values. The output is categorized as good, better and best. The values will be a real integer between 0 and 1 . IF - THEN rules are made out of the input parameters and based on this the output i.e. good, better, best sentences are taken out. Deffuzification of these fuzzy variables is done for getting the parameters into crisp value. Thus, the output will be produced. In the above table 2 , based on the importance of keywords, they are categorized as least, max, moderate. They are assigned a range of values between 0 to 1 .

Table 2. Values of keywords

\begin{tabular}{l|c|}
\hline FUZZY & CRISP INPUT \\
VARIABLE & VALUE OF KEYWORD \\
\hline LEAST IMPORTANT & $0-0.3$ \\
\hline MODERATELY IMPOTANT & $0.25-0.6$ \\
\hline MAXIMUM IMPORTANT & $0.55-1$ \\
\hline
\end{tabular}

Table 3. Values of location

\begin{tabular}{|l|c|}
\hline FUZZY & CRISP INPUT \\
VARIABLE & VALUE OF LOCATION \\
\hline MIDDLE & $0-0.3$ \\
\hline FIRST & $0.25-0.7$ \\
\hline LAST & $0.65-1$ \\
\hline
\end{tabular}

In the above table 3 , location of sentences is classified as first, middle and last. They are assigned a range between 0 and 1 on the basis of their position. In the above table 4, the output value of the sentences based on crisp value of keywords and location is assigned a range between 0 and 1 .
Table 3. Values of location

\begin{tabular}{|l|c|}
\hline $\begin{array}{l}\text { FUZZY } \\
\text { VARIABLE }\end{array}$ & CRISP INPUT \\
VALUE OF LOCATION
\end{tabular}

Table 4. Crisp output values

\begin{tabular}{|l|c|}
\hline $\begin{array}{l}\text { FUZZY } \\
\text { VARIABLE }\end{array}$ & $\begin{array}{c}\text { CRISP OUTPUT } \\
\text { VALUE }\end{array}$ \\
\hline GOOD & $0-0.2$ \\
\hline BETTER & $0.15-0.6$ \\
\hline BEST & $0.55-1$ \\
\hline
\end{tabular}

Figure 1: Sample Output for Automatic Text summarization for code mixed language

\section{Autematic Tet Sunmorice for Code-Mared langugage}

Automatic Text Summarizer for Code-Mixed Language Please Enter the Text Please Enter \% Of Summary

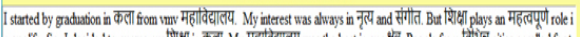

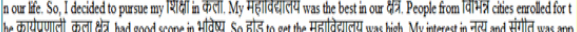

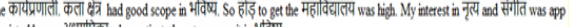
recinted by my अध्याप्येका who motrated me to pussue it in भीवेये.

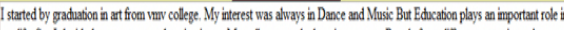

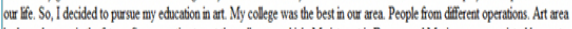
had good scope in the future. So competing to get the college was hiath My interest in Dances and Music was appreciated by my tea had good scope in the fiture Such

started by graduation in art from vurv college.My interest was always in Dance and Music But Educ ation plays an important role in our life.My college was the best in our area.Art area had good scope in the future.So competing to get the college was high.My interest in Dances and Music was appreciat ed by my teacher who foresees the funture.

\section{RESULTS AND DISCUSSION}

Compression ratio and retention ratio are two parameters for Evaluation of Summary. We have not used compression ratio as we are taking input for percentage of summary required from user. The text is evaluated using retention ratio as the parameter,

Retention Ratio: $R R=$ (No. of Sentences generated by System)/(No. of Sentences generated by Human)

We have tested our system for two domains Knowledge and Art. For each category five hundred documents of each category were tested. table 5 shows the sample of some documents from these two domains. Approximately our system retains $75 \%$ of Summary as compared to Human Expert. 
Figure 3: UV-VIS Spectra of the compound, PA-B-ester

\begin{tabular}{|c|c|c|c|c|c|c|c|}
\hline \multicolumn{4}{|c|}{ DOMAIN 1 - KNOWLEDGE } & & \multicolumn{3}{|c|}{ DOMAIN 2 - ART } \\
\hline $\begin{array}{l}\text { Sample } \\
\text { Docs }\end{array}$ & $\begin{array}{c}\text { System } \\
\text { generated } \\
\text { summary }\end{array}$ & $\begin{array}{c}\text { Human } \\
\text { generated } \\
\text { summary }\end{array}$ & $\begin{array}{l}\text { Retention } \\
\quad \text { ratio }\end{array}$ & $\begin{array}{c}\text { Sample } \\
\text { Docs }\end{array}$ & $\begin{array}{c}\text { System } \\
\text { generated } \\
\text { summary }\end{array}$ & $\begin{array}{c}\text { Human } \\
\text { generated } \\
\text { summary }\end{array}$ & $\begin{array}{l}\text { Retention } \\
\quad \text { ratio }\end{array}$ \\
\hline $\begin{array}{l}\text { Knowledge } \\
\text { 1.doc }\end{array}$ & 5 & 7 & $5 / 7$ & Art1.doc & 4 & 6 & $4 / 6$ \\
\hline $\begin{array}{l}\text { Knowledge } \\
\text { 2.doc }\end{array}$ & 4 & 6 & $4 / 6$ & Art2.doc & 7 & 9 & $7 / 9$ \\
\hline $\begin{array}{l}\text { Knowledge } \\
\text { 3.doc }\end{array}$ & 5 & 6 & $5 / 6$ & Art3.doc & 6 & 7 & $6 / 7$ \\
\hline \multicolumn{2}{|c|}{ RR for Domain-1 } & 0.738 & \multicolumn{3}{|c|}{ RR for Domain- } & & 0.767 \\
\hline
\end{tabular}

\section{CONCLUSION}

In this paper we represented different multilingual/ code mixed approaches of text summarization. The work of tokenization of words and sentences was done using Natural Language Toolkit. Translation of source and destination language has been done using GoogleTrans API. Various translation methods were studied like goslate, anglabharti, Moses. Tkinter was used for making graphical user interface. Summarization was done using Fuzzy Logic. We have used location and keywords as input values. In future more, more input values can be taken for better results and more intelligent methods can be applied.

\section{REFERENCES}

Ansamma John, M Wilscy. "Vertex Cover Algorithm Based Multi-Document Summarization using information content of sentences", ScienceDirect, Procedia Computer Science 46, 2015, pp 285 - 292.

Atif Khan, Naomie Saleem, "A review on abstractive summarization methods", Faculty of Computing, Johor, Malaysia. Journal of Theoretical and Applied Information Technology, Vol. 59 No.1, 2016, 64-72.

Ben King, Steven Abney, Labeling the languages of words in mixed-language documents using weakly supervised methods. In Proceedings of NAACL-HLT, 2013, pages 1110-1119.

F. Kiyoumarsi, H. Khoshravi, E. Eslami, M. Davoudi, "Extraction based Text Summarization using Fuzzy Analysis”, Iranian journal of fuzzy systems, 2010.

Ganpathiraju, M.K., Carbonell, J. Yang, Y., "Relevance of cluster size in MMR based Summarizer: A report”, 2002, 11-742.

G. Rohini, P. Krishna Subba Rao, Ch. Avinash. "Textsum: An automatic text summarization tool using Bayesian classification”, Recent trends in Programming Language,
2014, Vol 1, No 1.

Madhuri. A. Tayal, M. M. Raghuwanshi, and L. G. Malik, "Identification of Title for Natural Languages using, Resource Description Framework and Anaphoric Reference Resolution”. Springer: Communications in Computer and Information Science (CCIS), Volume 276, , 2013, PP 75-88.

Madhuri. A. Tayal, M. M. Raghuwanshi, and Animesh Tayal, "SUMTOD: summarization of toddler stories through sentence Reduction and sentence combination,", International Journal of Advanced Research in Computer Science, Volume 9, No. 1, 2018, pp 229-235.

Madhuri. A. Tayal, M. M. Raghuwanshi, and L. G. Malik, "Syntax Parsing: Implementation using GrammarRules for English Language”, IEEE. International Conference on Electronic Systems, Signal Processing and Computing Technologies, IEEE, 2014, pp. 376-381. ACM Digital Library 14117501.

Madhuri A. Tayal, M. M. Raghuwanshi, and L. G. Malik, "ATSSC: Development of an approach based on soft computing for text summarization," Elsevier, Science Direct journal: Computer Speech Language, Vol. 41, 2017, pp. 214-235.

Madhuri. A. Tayal, M. M. Raghuwanshi, and L. G. Malik, Word net-based Method for Determining Semantic Sentence Similarity through various Word Senses, ICON 2014, ACL Anthology, 2014, 397-403.

R.M.K. Sinha, K. Sivaraman, A. Agrawal, R. Jain, R. Srivastava, A. Jain, "ANGLABHARTI: a multilingual machine aided translation project on translation from English to Indian languages", Systems, man and cybernetics, IEEE, 1995. vol 5.

Rada Mihalcea, Paul Tarau, "Textrank: Bringing order into texts", Proceedings of the 2004 Conference on Empirical Methods in Natural Language Processing, 
EMNLP -04, 2004, ACL Anthology.

Spandana gella, Kalika bali, monojit Choudhary. "ye word kis lang ka hai bhai?” Testing the limits of word level language identification. university of edinburg Microsoft research lab, India, 2014.

Tanveer Siddiqui, U.S. Tiwary, "Natural Language
Processing and Information Retrieval", Oxford University Press.

U. Ahmed, K. Bali, M. Choudhury, and V. B. Sowmya, "Challenges in designing input method editors for Indian languages: The role of word-origin and context". In Proceedings of IJCNLP Workshop on Advances in Text Input Methods, 2011. 